Canad. Math. Bull. Vol. 23 (4), 1980

\title{
A THEOREM ON THE DENSENESS OF ORBITS IN METRIC SPACES
}

\author{
BY \\ ABRAHAM BOYARSKY* AND MANNY SCAROWKSY \\ Abstract. Let $\tau$ be a transformation from a compact metric \\ space $X$ into itself. Conditions are presented which ensure that there \\ exists an orbit $\left\{\tau^{n}(x)\right\}_{n=1}^{\infty}$ which is dense in $X$. An example is given.
}

1. Introduction. Let $X$ be a compact metric space and $\tau$ a mapping from $X$ into $X$. If $X$ is an interval of the real line, sufficient conditions are known [1] which ensure the existence of a point $x \in X$ such that $\left\{\tau^{n}(x)\right\}_{n=0}^{\infty}$ is dense in $X$, where $\tau^{n}=\tau \circ \tau^{n-1}$ is the $n$th iterate of $\tau$. The aim of this note is to extend this result to a general compact metric space. In $[2,3,6,7]$ the existence of invariant measures is established for expanding maps. In $[2,3,6]$ the underlying space is restricted to a compact, connected smooth manifold. These existence results do not say anything about the supports of the invariant measures. The existence of dense orbits in a subset $\bar{X} \subset X$ would be helpful in locating the support of the invariant measure. In [5], conditions are presented which guarantee the existence of orbits that do not approach any periodic pattern, but in themselves they do not establish denseness even in a subset of the underlying space.

2. Denseness of orbit. Let $(X, \rho)$ be a compact metric space, and let $\left\{X_{1}, X_{2}, \ldots, X_{n}\right\}$ be a finite partition of $X$ such that each $X_{i}$ is closed and compact. Let $H=\bigcup_{i \neq j}\left(X_{i} \cap X_{j}\right)$ denote the boundary of the partition. Let $\tau: X \rightarrow X$ be a mapping such that $\left.\tau\right|_{X_{i}}$ is continuous. Define $B=\bigcup_{n=0}^{\infty} \tau^{-n}(H)$.

Theorem. Let $(X, \rho)$ and $\tau$ be as above. Assume $B \neq X$ and (1) $\left.\tau\right|_{X_{i}}$ is expanding for $i=1, \ldots, n$, i.e., there exist $d_{i}>1$ such that $\rho(\tau(x), \tau(y))>$ $d_{i} \rho(x, y)$ for every $x, y \in X_{i}$.

(2) for each pair $(i, j), i, j \in(1, \ldots, n)$, there exists an integer $n_{i j} \ni \tau^{n_{i j}}\left(X_{i}\right) \supset X_{i}$

(3) for each $i$ there exist integers $n_{i}, l_{i} \in(1, \ldots, n) \ni \tau\left(X_{i}\right)=\bigcup_{j=l_{i}}^{n_{i}} X_{j}$. Then there is a point $x \in X$ such that the orbit $\left\{\tau^{n}(x)\right\}_{n=0}^{\infty}$ starting at $x$ is dense in $X$.

Proof. Associate with each $x \notin B$ the coding $\langle x\rangle$ of $x$ as follows:

$$
\langle x\rangle=. i_{1} i_{2} i_{3} \ldots \ldots \ldots
$$

Received by the editors March 2, 1979 and in revised form August 8, 1979.

* The research of this author was supported by a grant from the National Research Council of Canada: Grant No. A-9072. 
where $x \in X_{i_{1}}, \tau(x) \in X_{i_{2}}, \tau^{2}(x) \in X_{i_{3}}, \ldots \ldots$ This coding is well-defined since we cannot have $\tau^{i}(x) \in X_{k} \cap X_{l}$. Further, we claim:

(i) if two codings are equal, i.e., $\langle x\rangle=\langle y\rangle$, then $x=y$, and

(ii) if.$i_{1} i_{2} i_{3} \ldots$ is a coding and $\tau$ satisfies $\tau\left(X_{i_{j}}\right) \supset X_{i_{i+1}}, j=1,2,3, \ldots$ (we refer to such a coding as allowable), then there exists a unique $x \in X$ such that $\langle x\rangle=. i_{1} i_{2} i_{3} \ldots$

To prove (i), we note that our hypotheses implies $\rho\left(\tau^{n}(x), \tau^{n}(y)\right) \geq d^{n} \rho(x, y)$, where $d=\min \left(d_{1}, d_{2}, \ldots, d_{n}\right)$. Since $d>1$ and $\rho$ is bounded on $X \times X$, this gives a contradiction unless $x=y$.

To prove (ii), we define

$$
J_{k}=\left\{x: x \in X_{i_{1}}, \tau(x) \in X_{i_{2}}, \ldots, \tau^{k-1}(x) \in X_{i_{k}}\right\}
$$

We note that $\left\{J_{k}\right\}_{k=1}^{\infty}$ is a collection of compact sets with the finite intersection property. Thus $\bigcap_{k>1} J_{k}$ is non-empty and by (i) has no more than one element.

We can now associate allowable codings in a $1-1$ and onto fashion with $x \in X-B$. To prove the existence of a dense orbit, form the set of finite allowable sequences

$$
\left\{i_{k}, i_{k} i_{l}, i_{k} i_{l} i_{m}, \ldots\right\}
$$

where, for example, the symbol $i_{k} i_{l}$ represents all sequences $i_{k} i_{l}$ such that $\tau\left(X_{i_{k}}\right) \supset X_{i_{1}}$. These are countable. Let $S_{1}, S_{2}, S_{3}, \ldots$ be an enumeration of them. Now form the coding . $S_{1} T_{1} S_{2} T_{2} S_{3} T_{3} \ldots$, where the $T_{i}$ are inserted to make this an allowable sequence. This is permitted by hypothesis (2). Let the coding represent the point $x \in X$. Let $y \in X$ and $\varepsilon>0$ be given. We want to show that there exists an integer $n$ such that $\left|\tau^{n}(x)-y\right|<\varepsilon$. Let the symbol $S_{p}$ correspond to the coding of $\left\{y, \tau(y), \ldots, \tau^{m}(y)\right\}$, i.e., if $y \in X_{i_{1}}, \ldots, \tau^{m}(y) \in X_{i_{m}}$, then $S_{p}=i_{1} \ldots i_{m}$. We then have:

$$
\begin{array}{cc}
\tau^{p}(x) \in X_{i_{1}}, & y \in X_{i_{1}} \\
\tau^{p+1}(x) \in X_{i_{2}}, & \tau(y) \in X_{i_{2}} \\
\cdot & \\
\cdot & \\
\tau^{p+m}(x) \in X_{i_{m}}, & \tau^{m}(y) \in X_{i_{m}}
\end{array}
$$

By the expanding property of $\tau$,

$$
\left|\tau^{p}(x)-y\right|<d^{-m}\left|\tau^{p+m}(x)-\tau^{m}(y)\right|<\varepsilon,
$$

if $m$ is chosen sufficiently large. This proves the denseness of the orbit $\left\{\tau^{n}(x)\right\}_{n=0}^{\infty}$ in $X$.

We note that the theorem does not require $\tau$ to be non-singular. If it is and $X$ is an $n$-dimensional space, then $B \neq X$ is implied by the condition $m(H)=0$, where $m$ is the Lebesgue measure. 
3. An example. Let $\tau:[0,1] \times[0,1] \rightarrow[0,1] \times[0,1]$ be defined by $\tau(x, y)=$ $(f(x), f(y))$, where

$$
f(t)=\left\{\begin{array}{cc}
2 t & 0 \leq t \leq \frac{1}{2} \\
2-2 t, & \frac{1}{2} \leq t \leq 1
\end{array}\right.
$$

Let $\quad X_{1}=\left[0, \frac{1}{2}\right] \times\left[0, \frac{1}{2}\right], \quad X_{2}=\left[0, \frac{1}{2}\right] \times\left[\frac{1}{2}, 1\right], \quad X_{3}=\left[\frac{1}{2}, 1\right] \times\left[0, \frac{1}{2}\right], \quad$ and $\quad X_{4}=$ $\left[\frac{1}{2}, 1\right] \times\left[\frac{1}{2}, 1\right]$. It is easy to show that $\tau$ and the partition $\left\{X_{1}, X_{2}, X_{3}, X_{4}\right\}$ satisfy the conditions of the theorem. Hence there exists an orbit $\left\{\tau^{n}(x)\right\}_{n=0}^{\infty}$ which is dense in $[0,1] \times[0,1]$. The one dimensional version of this result is known [4].

\title{
REFERENCES
}

1. A. Boyarsky and M. Scarowsky, On a class of transformations which have unique absolutely continuous invariant measures, Trans. Amer. Math. Soc. 255, (1979) 243-262.

2. M. A. Avez, Propriétes ergodiques des endomorphismes dilatants des varieté compactes, C.R. Acad. Sc. Paris, Serie A, t. 266, (1968) 610-612.

3. K. Krzyzewski and W. Szlenk, On invariant measures for expanding differentiable mappings, Studia Mathematica, T. XXXIII, (1962) 83-92.

4. J. Guckenheimer, G. Oster, and A. Jpaktchi, The dynamics of denisty dependent population models, J. Math. Biol., 4, (1977) 101-147.

5. F. R. Marotto, Snap-back repellers imply chaos in $R^{n}$, Jour. Math. Anal. and Appl. 63, (1978) 199-223.

6. P. Walters, Invariant measures and equilibrium states for some mappings which expand distances, Trans. Amer. Math. Soc. 236, (1978) 121-153.

7. A. Lasota and J. A. Yorke, On the existence of invariant measures for piecewise monotonic transformations, Trans. Amer. Math. Soc. 186, (1973) 481-488.

\author{
DePaRTMENT OF MATHEMATICS \\ Sir George Williams Campus \\ CONCORDIA UNIVERSITY \\ Montreal, CANAdA
}

